\title{
EVERY UNCOUNTABLE ABELIAN GROUP ADMITS A NONNORMAL GROUP TOPOLOGY
}

\author{
F. JAVIER TRIGOS-ARRIETA
}

(Communicated by Franklin D. Tall)

\begin{abstract}
If $\mathbf{G}$ is a locally compact Abelian group, let $\mathbf{G}^{+}$denote the underlying group of $\mathbf{G}$ equipped with the weakest topology that makes all the continuous characters of $\mathbf{G}$ continuous. Thus defined, $\mathbf{G}^{+}$is a totally bounded topological group. We prove:
\end{abstract}

Theorem. $\mathbf{G}^{+}$is normal if and only if $\mathbf{G}$ is $\sigma$-compact.

When $\mathbf{G}$ is discrete, this theorem answers in the negative a question posed in 1990 by E. van Douwen, and it partially solves a problem posed in 1945 by A. Markov.

\section{INTRODUCTION}

All spaces are completely regular and Hausdorff, and all groups are Abelian.

For a locally compact Abelian group $\mathbf{G}$ define $\mathbf{G}^{+}$as in the abstract. The topology of $\mathbf{G}^{+}$is known as the weak topology on $\mathbf{G}$ and has been studied, among others, by Glicksberg in [4] and by Trigos-Arrieta in [10, 11]. In his 1990 paper E. van Douwen studied the space $\mathbf{G}^{+}$for $\mathbf{G}$ discrete, i.e., $\mathbf{G}$ equipped with its maximal totally bounded (topological group) topology, and asked whether this topology was normal when $|\mathbf{G}|>\omega$.

On the other hand, A. Markov, back in 1945, asked whether every uncountable group-Abelian or not-would admit a nonnormal Hausdorff topological group topology. Without offering any details or citing the existence of a manuscript in preparation, Markov attributed to E. Livenson a proof that every uncountable Abelian group admits such a topology. Despite the effort of many mathematicians, it has been impossible to determine the basis of this attribution and the nature of Levinson's presumed construction. It is clear that by considering the maximal totally bounded topology, the negative answer to van Douwen's question provides a positive answer to Markov's problem in the Abelian situation. Those interested in the nonabelian case are referred to the informative survey [1].

Received by the editors August 17, 1992 and, in revised form, February 11, 1993; presented by the author at the Eighth Summer Conference on Topology \& Applications, held in Flushing, NY, in June 1992.

1991 Mathematics Subject Classification. Primary 22B05, 54A10, 54A25, 54H11.

Key words and phrases. Normal space, independent set, Bohr compactification, totally bounded topology. 


\section{THE RESULT}

Our strategy is to deal first with the situation when $\mathbf{G}$ is discrete, leaving the general case as a corollary.

A subset $\Lambda$ of an Abelian group $\mathbf{G}$ is said to be independent if $0 \notin \Lambda$, and for every choice $\left\{a_{i}\right\}_{i=1}^{n}$ from $\Lambda$ and $\left\{m_{i}\right\}_{i=1}^{n}$ of integers, the equality $m_{1} a_{1}+\cdots+m_{n} a_{n}=0$ implies $m_{i} a_{i}=0$ for all $i$. See $[3, \S 16]$.

As noticed in $[5,(1.4)]$, independent subsets of $\mathbf{G}$ are always closed and discrete in $\mathbf{G}^{+}$.

If $\mathbf{S}$ is a subset of a space $\mathbf{X}$, we denote by $\mathrm{Cl}_{\mathbf{X}} \mathbf{S}$ the closure of $\mathbf{S}$ in $\mathbf{X}$. If $\mathbf{S}$ is a subset of a group $\mathbf{G}$, we denote by $\langle\mathbf{S}\rangle$ the subgroup of $\mathbf{G}$ generated by $\mathbf{S} . \quad \beta \mathbf{X}$ and $\mathbf{b G}$ will denote the Čech-Stone compactification of $\mathbf{X}$, and the Bohr compactification of $\mathbf{G}$, respectively. The main result of this article follows. The style of proof here is due to the referee, based on the author's arguments; see Trigos-Arrieta [12].

Theorem. If $\mathbf{G}$ is a (discrete) Abelian group, then $|\mathbf{G}|>\omega$ if and only if $\mathbf{G}^{+}$is not normal.

Proof. $(\Rightarrow)$ Let $\mathbf{G}$ be an uncountable group. By $[3, \S 16]$ or [7] we can take an uncountable independent subset $\Lambda$ of $\mathbf{G}$. By [5, (1.4)] and [2, (1.13(a))] we can assume that any subset of $\Lambda$ is $\mathbf{C}^{*}$-embedded in $\mathbf{b G}$. Divide $\Lambda$ into two subsets $\Lambda_{1}$ and $\Lambda_{2}$ of the same cardinality, and let $\mathbf{H}_{1}=\left\langle\Lambda_{1}\right\rangle$ and $\mathbf{H}_{2}=\left\langle\Lambda_{2}\right\rangle$. Clearly $\mathrm{Cl}_{\mathbf{b H}_{1}} \Lambda_{1}=\beta \Lambda_{1}$ and $\mathrm{Cl}_{\mathbf{b H}_{2}} \Lambda_{2}=\beta \Lambda_{2}$. This implies that $(\beta \Lambda)^{2} \subseteq \mathbf{b} \mathbf{H}_{1} \times \mathbf{b} \mathbf{H}_{2} \subseteq$ bG. If $\Delta:=\{(\lambda, \lambda): \lambda \in \Lambda\}$, we note as well that $\Delta$ and $\Phi:=\Lambda^{2} \backslash \Delta$ are closed and discrete subsets of $\mathbf{G}^{+}$with $\Delta \cap \Phi=\varnothing$. One must show that $\Delta$ and $\Phi$ cannot be separated by open sets in $\mathbf{G}^{+}$. Let $\mathbf{U}$ be an arbitrary open set in $\mathbf{G}^{+}$containing $\Phi$. By $\left[9,1.5\right.$ and 1.6] $\mathbf{F}:=\mathrm{Cl}_{\mathbf{b G}} \mathbf{U}$ is a zero-set of $\mathbf{b G}$. Since $(\beta \Lambda)^{2} \backslash \mathbf{F}$ is Lindelöf (being cozero in $\left.(\beta \Lambda)^{2}\right)$ and $\Delta$ is discrete and open in $(\beta \Lambda)^{2}$, it follows (by considering for each $p \in(\beta \Lambda)^{2} \backslash \mathbf{F}$ an open (in $\left.(\beta \Lambda)^{2}\right)$ rectangle $R_{p} \subseteq(\beta \Lambda)^{2} \backslash \mathbf{F}$, if necessary) that $(\beta \Lambda)^{2} \backslash \mathbf{F}$ is at most countable. Therefore, $\Delta \cap \mathbf{F} \neq \varnothing$, implying that $\Delta \cap \mathrm{Cl}_{\mathbf{G}^{+}} \mathbf{U} \neq \varnothing$. If $\mathbf{V}$ were an open set in $\mathbf{G}^{+}$such that $\Delta \subseteq \mathbf{V}$ and $\mathbf{U} \cap \mathbf{V}=\varnothing$, we would have $\Delta \cap \mathrm{Cl}_{\mathbf{G}^{+}} \mathbf{U}=\varnothing$. This contradiction proves $(\Rightarrow)$.

$(\Leftarrow)$ Obvious.

For the general case we can proceed as follows: Clearly the weak topology on a locally compact $\sigma$-compact Abelian group must be normal. Let $\mathbf{G}$ be a locally compact Abelian group which is not $\sigma$-compact. By [6, (24.30)] $\mathbf{G}$ is topologically isomorphic to $\mathbf{R}^{n} \times \mathbf{G}_{0}$, where $\mathbf{R}$ denotes the real line, $n$ is a nonnegative integer, and $\mathbf{G}_{0}$ has a compact open subgroup $\mathbf{H}_{0}$. Apply the theorem to the uncountable discrete group $\mathbf{G}_{0} / \mathbf{H}_{0}$ and use $[10,(2.2) ; 11, \S 3 ; 6$, (5.18)]. Thus we have

Corollary. If $\mathbf{G}$ is a locally compact Abelian group, then $\mathbf{G}$ is $\sigma$-compact if and only if $\mathbf{G}^{+}$is normal.

Remark. The paper [12] deals with the same results of this article in greater detail. 


\section{ACKNOWLEDGMENT}

Special thanks to my teacher Wis Comfort for the valuable conversations concerning the main result of this article. I am also grateful to Tony Hager, Sophia U. Raczkowski-Trigos, Lew Robertson, and Jan van Mill for advising me.

\section{REFERENCES}

1. W. W. Comfort, K. H. Hofmann, and D. Remus, Recent developments in topological groups and topological semigroups, Recent Progress in General Topology (M. Husek and Jan van Mill, eds.), North-Holland, Amsterdam, 1992, pp. 57-144.

2. E. van Douwen, The maximal totally bounded group topology on $G$ and the biggest minimal G-space, for Abelian groups $G$, Topology Appl. 34 (1991), 69-91.

3. L. Fuchs, Infinite Abelian groups, Vol. I, Pure Appl. Math., vol. 36, Academic Press, New York and London, 1970.

4. I. Glicksberg, Uniform boundedness on groups, Canad. J. Math. 14 (1962), 269-276.

5. K. P. Hart and J. van Mill, Discrete sets and the maximal totally bounded group topology, J. Pure Appl. Algebra 70 (1991), 73-80.

6. E. Hewitt and K. A. Ross, Abstract harmonic analysis, Vol. I, Grundlehren Math. Wiss., vol. 115, Springer-Verlag, Berlin, Göttingen, and Heidelberg, 1963.

7. L. J. Kulikov, On the theory of Abelian groups of arbitrary cardinality, Mat. Sb. 9 (1941), 517-547. (Russian)

8. A. A. Markov, On free topological groups, Izv. Akad. Nauk SSSR Ser. Math. 9 (1945), 3-64; English transl., Amer. Math. Soc. Transl. Ser. 1, vol. 8, Amer. Math. Soc., Providence, RI, 1962, pp. 195-272.

9. K. A. Ross and K. R. Stromberg, Baire sets and Baire measures, Ark. Mat. 6 (1967), 151-160.

10. F. J. Trigos-Arrieta, Continuity, boundedness, connectedness and the Lindelöf property for topological groups, J. Pure Appl. Algebra 70 (1991), 199-210.

11. __ Pseudocompactness on groups, General topology and its applications, Fifth Northeast Conference, Marcel Dekker, New York, 1991, pp. 369-378.

12. __ Normality and the weak topology on a locally compact Abelian group, Proc. Eighth Summer Topology Conference, Ann. New York Acad. Sci. (to appear).

Department of Mathematics, California State University, Bakersfield, 9011 Stockdale Highway, Bakersfield, California 93311-1099

E-mail address: jtrigos@csbina.csubak.edu 